\title{
MÉDIAS ET LANGUES NATIONALES AU BURKINA FASO
}

\author{
Serge Théophile BALIMA ${ }^{1}$
}

En dehors de quelques pays, l'introduction des langues locales dans les médias est intervenue en Afrique, avec le processus irréversible de décolonisation, après la seconde guerre mondiale. Durant cette époque des années 50, le paradigme de la modernisation a dominé les théories du développement. Il prétend essentiellement que le développement consiste en un processus de rattrapage impliquant la modernisation des pays du Sud sur le modèle de ceux du Nord. Ainsi, le développement était évalué d'abord en terme d'accroissement de la productivité des pays du Sud qui, pour ce faire, doivent adopter les connaissances, les techniques et les technologies du Nord.

En vertu de ce paradigme, les cultures traditionnelles des pays africains sont considérées, en grande partie, comme la cause des problèmes de développement auxquels ils sont confrontés. Par conséquent, pour qu'un pays avance sur la voie du développement, les populations se doivent d'adopter des comportements plus modernes et d'abandonner progressivement leur culture traditionnelle. Et compte tenu de l'impor-

1 Directeur du Centre d'Expertise et de Recherche africain sur les Médias et la Communication (CERAM), Université de Ouagadougou.

Recherches en communication, $\mathrm{n}^{\circ} 24$ (2005). 
tance accordée aux idées, à la culture et aux mœurs dans ce paradigme de la modernisation à l'occidentale, on ne peut s'étonner que la notion d'information y joue un rôle prépondérant. En effet, le diagnostic établi par les tenants de cette approche prétend que la persistance de pratiques, de conceptions et d'attitudes des sociétés traditionnelles serait attribuable au manque d'information. Les médias doivent jouer un rôle stratégique dans la diffusion et l'implantation d'idées, d'attitudes et de comportements plus modernes.

C'est dans un tel contexte que la plupart des langues ont été introduites dans la propagation de l'Evangile et dans les programmes radiophoniques essentiellement. A cette époque, l'étude de la communication se définissait comme une étude de la persuasion, comme une munition magique capable de provoquer des changements de comportements au sein des populations.

\section{L'avènement des langues nationales dans l'audiovisuel}

En introduisant donc les langues nationales dans les médias, il s'agissait de promouvoir les conseils sur la santé, l'hygiène publique, la protection de l'environnement, la scolarisation et la vaccination des enfants etc. L'utilisation des langues locales permettrait une meilleure circulation du flux d'information depuis l'émetteur institutionnel jusqu'aux récepteurs dans leurs diversités. Ainsi, le nombre de langues utilisées sur les antennes de la Radio Nationale est allé croissant de 1960 à nos jours. Déjà à la fin des années 70, Radio Ouaga émettait en 18 langues nationales qui n'occupaient au total que moins de $20 \%$ du volume horaire total d'émission. Progressivement, la nécessité de développer les nouvelles d'actualité en langues nationales apparut avec les plans quinquennaux de « développement économique ». Il s'agissait de permettre aux populations rurales non encore alphabétisées et peu portées vers la modernité d'intégrer les nouvelles méthodes de cultures, d'assimiler les recommandations gouvernementales et d'exécuter les mots d'ordre du pouvoir central.

Ce déséquilibre dans la répartition des heures d'émission est la résultante d'un rapport de force politico -culturelle qui tend à ravaler les langues du terroir au rang d'outils de seconde zone. Ce constat est assez répandu en Afrique francophone où, généralement, les langues locales conservent un statut inférieur par rapport à la langue officielle.

Le volume général des programmes audiovisuels en langues nationales croît régulièrement à une échelle nettement inférieure par rapport 
au français qui reste la langue dominante de la radio et de la télévision depuis l'indépendance. Cette évolution s'est faite en quatre phases qui correspondent à quatre périodes socio-historiques du Burkina Faso.

La première se situe entre 1959 et 1962 où le nombre de langues nationales utilisées augmente considérablement à la radio. Trois langues en 1959, mooré, jula et fulfuldé puis apparition du gourounsi en 1960, utilisation du lobi, du bobo et du sénoufo en 1961, irruption du bissa et du samo en $1962 . .$.

La deuxième phase se situe entre 1966 et 1979 qui voit surgir huit autres langues nationales dans la diffusion des programmes radiophoniques. Pendant ce temps, la télévision, toujours embryonnaire, ne recourt qu'à deux ou trois langues, les plus répandues de toute évidence, et n'en modifiera pas le nombre jusqu'à l'avènement de la Révolution du 4 août 1983.

La troisième phase caractérisée par la réaffirmation de l'identité culturelle nationale met en relief l'usage des langues locales comme moyen de mobilisation, d'éducation et de conscientisation des masses. Cette période, ouverte en 1983, débouche sur les ambitions révolutionnaires dans le système d'information mais l'immaturité et le spontanéisme de certains acteurs ont dévoyé les bonnes intentions des leaders politiques.

La quatrième phase coïncide avec l'avènement de la $\mathrm{IV}^{\mathrm{e}}$ République qui, grâce à l'option libérale du régime, verra fleurir des initiatives privées et une liberté de ton dans les productions médiatiques. Cette époque, ouverte depuis une quinzaine d'années, a été aussi celle de la multiplication des langues locales sur les antennes de l'audiovisuel, en particulier la radio. Celle-ci connaît, en effet, son début de printemps avec aujourd'hui plus de soixante radios privées commerciales, communautaires et associatives. Parmi ces radios, les trois quarts utilisent suffisamment les langues locales en fonction des parlers locaux ou régionaux.

Cependant, cette importance numérique des langues du terroir ne traduit pas un rapport de force véritable en faveur de leurs locuteurs, c'est-à-dire ceux qui n'utilisent quotidiennement et exclusivement que les parlers locaux. Le fond de la question est de savoir quel type d'émission est diffusé dans ces différentes langues du terroir.

Une analyse comparative effectuée en 1979 sur les répartitions catégorielles des programmes radiophoniques en langue française et en langues locales révèle une stagnation des rubriques d' "information » en langues autochtones (15\%) de 1961 à 1979, et ce, en dépit de l'ac- 
croissement du nombre de ces langues sur les antennes ${ }^{1}$. Il en est de même pour les programmes dits «éducatifs »; alors que l'urgence du développement commande un réajustement de ces répartitions. Le seul terrain où le français semble faire des concessions est celui du divertissement. En effet, dans ce domaine, la langue officielle recule sensiblement parce que l'émergence des stations privées de toute nature a entraîné l'accroissement des musiques, contes et animations récréatives en langues du pays, au détriment, hélas, de programmes vraiment socioéducatifs.

En évoquant le cas de son pays, Moustapha Samb du Sénégal, dresse lui aussi un triste constat sur l'utilisation des langues locales sur les ondes durant les décennies passées. «Pendant longtemps donc, l'information régionale en langues nationales est restée à la traîne ; elle a joué une partition démodée que les populations n'ont pas su apprécier. Elle n'a pas réussi à se débarrasser de sa mission classique pour s'intéresser davantage aux préoccupations quotidiennes des populations ${ }^{2}$.

Pourr sa part, Serge Théophile Balima analysant en 1976 les Programmes radiophoniques et systèmes culturels en Haute-Volta affirmait que « le public du village attend de la radio qu'elle parle un certain langage, qu'elle aborde un certain sujet dont il peut cerner la réalité $3 . »$ Car effectivement, comment peut-on cerner cette réalité quand les programmes diffusés ne prolongent pas leur univers culturel ? «Mais l'usage d'une langue africaine est une réconciliation, le fait que le Mossi ou le Peul ou encore le Wolof écoute sa propre langue à la télévision ou à la radio crée un rapport de transparence. Cette communication lui apporte confiance non seulement dans l'outil qui cesse d'être distant, mais dans sa propre langue ${ }^{4} \gg$. En fait l'usage des langues locales permet de donner une force au discours radiophonique auprès des populations qui sont d'ailleurs toujours fières d'entendre les langues de leurs ancêtres à la radio.

L'utilisation des langues nationales dans les médias de grande diffusion constitue un facteur de participation des populations non seulement pour le développement, mais pour l'émergence d'une conscience iden-

1 Serge Théophile Balima, Programmes radiophoniques et système culturel en Haute Volta, mémoire maîtrise en Journalisme, Université de Bordeaux III, 1977.

2 Moustapha Samb. Médias, décentralisation et langues nationales: les voix d'une nouvelles citoyenneté. Médias@actions, n³3, janvier -mars 2003, pp.10-17.

3 S. T. Balima, ibid.

4 Moustapha Samb, Ibid. 
titaire et citoyenne. Au regard du fort taux d'analphabétisme qui frappe les populations rurales et urbaines, l'audiovisuel apparaît comme le moyen le plus approprié pour établir une vraie communication entre pouvoirs publics et communautés linguistiques du pays.

C'est l'une des voies de solution pour rendre la parole aux populations et aboutir rapidement à une démocratisation de l'expression populaire. En ce sens, l'émergence des radios communautaires et associatives dans l'espace national burkinabè peut être considérée comme le début d'une liberté trouvée ou retrouvée : celle d'entendre ce qui vient d'autrui et de se faire entendre par autrui.

A travers les langues locales parlées à la radio, c'est donc tout le potentiel régional ou identitaire qui est ainsi valorisé : les compétences locales s'affirment dans la parole libérée tandis que les langues locales deviennent le vecteur d'une renaissance culturelle prometteuse.

En quelques années de présence ou d'omniprésence des langues locales dans les programmes des radios locales, les résultats ne sont pas négligeables, tant sur le plan de la reconnaissance identitaire que sur celui de la valorisation du patrimoine culturel.

En quelques années, ces radios de proximité ont situé les langues nationales à un niveau que les décennies de lutte politique pour leur revalorisation n'ont permis d'atteindre. En somme, ce que l'utilisation des langues nationales a permis de faire avancer dans le domaine du développement local est sans commune mesure avec l'impact du français qui reste la langue d'une élite minoritaire, occidentalisée voire marginalisée.

\section{L'avènement des langues dans la presse écrite}

Il existe une presse écrite en langues nationales au Burkina Faso. Son existence apparaît comme l'antidote de la centralité de la presse écrite; encore que sur 23 journaux en langues nationales, plus d'une dizaine sont édités à Ouagadougou. A signaler que le seul journal en langues nationales, le Partenaire (en jula: Demenegon) édité à BoboDioulasso, ne paraît plus. En fait, par delà Bobo-dioulasso, c'est tout l'Ouest-Burkina qui ne dispose pas de journal en langues nationales de publication régulière, en dehors de quelques organes de liaison à caractère évangélique.

La presse écrite en langues nationales a profité de l'environnement politique favorable après 1987 pour se diversifier à l'échelle natio- 
nale. Certes, Hakilifalen ( «L'Echange», en jula), édité par l'INADESFormation Ouagdougou, est censé être distribué dans cette zone. Mais on connaît bien le caractère aléatoire de la diffusion de la presse écrite burkinabè auquel la plupart des journaux en langues nationales n'échappe pas.

En une décennie d'actions, les journaux en langues nationales ont contribué à la formation d'un environnement lettré en milieu paysan et à la promotion des langues nationales. Certains des titres (Sôore, Laabaali, etc.) sont devenus des instruments d'alphabétisation et de post-alphabétisation. Les acteurs de l'alphabétisation, Directions provinciales de l'enseignement de base, Ministère de l'agriculture en sont contents et fiers. Ces titres sont très appréciés par les organisations paysannes et réclamés par les producteurs qui ont, à travers leurs colonnes, un tremplin pour promouvoir, non seulement leurs activités, mais aussi pour se faire connaître et communiquer entre eux.

Ces journaux forment les producteurs dans les domaines de l'agriculture, de l'élevage, de la protection de l'environnement et de la santé. Cette dernière est même devenue l'une des rubriques prisées (aussi bien des lecteurs de Sôore que de ceux de Laabaali que nous avons pu visiter). Dans certains cas, ils constituent le ciment identitaire provincial ou régional.

Par leurs actions, les paysans des zones couvertes ont pu, par ailleurs, s'ouvrir à la conscience régionale, nationale et internationale. Le nombre de rubriques dans un titre tel Sôore est explicite, il couvre des domaines très variés :

$\begin{array}{ll}\text { - Actualité } & \text { - Culture générale } \\ \text { - Etranger } & - \text { Politique } \\ \text { - Sport } & \text { - Coutume } \\ \text { - Drames } & \text { - Editorial } \\ \text { - Alphabétisation } & \text { - Développement } \\ \text { - Agriculture et élevage } & - \text { Femmes } \\ \text { - Gestion de l'environnement } & \text { - Eveil à la connaissance } \\ \text { - Changement de mentalité } & - \text { Pensée }\end{array}$

Grâce aux journaux en langues nationales, les paysans alphabétisés peuvent s'exprimer. Du reste, la plupart de ces titres sont écrits par des paysans alphabétisés. Par exemple, 62,3\% des articles de Sôore sont écrits par les lecteurs (même si ces articles se concentrent sur une 
seule page et portent essentiellement sur les problèmes et conseils dans le domaine de la santé) contre $31,7 \%$ par les rédacteurs.

Les contenus des journaux en langues nationales sont assez bons, si l'on tient compte généralement des équipes de rédaction qui ne comportent pas un seul professionnel. Ces contenus peuvent encore être améliorés, pour peu que soient organisées, pour les rédacteurs, des sessions annuelles d'initiation à l'écriture journalistique et aux techniques du secrétariat de rédaction. La formation du monteur de Laabaali sur PAO (publication assistée par ordinateur), a permis à ce dernier de produire des contenus intéressants qui placent ce titre parmi les mieux présentés des journaux en langues nationales.

L'initiation à l'écriture journalistique est également une nécessité pour les correspondants. Sur ce point, un titre comme Laabaali est dans la bonne direction, d'abord parce qu'il recrute ses correspondants sur test, et ensuite parce qu'il organise, chaque année, des sessions de formation pour eux (cette formation est assurée, entre autres, par le correspondant local de l'Agence d'information du Burkina (AIB)).

Enfin, les journaux en langues nationales ont un taux de pénétration très intéressant dans les campagnes. Sôore, par exemple, quadrille tous les vingt et un départements de l'ancien Yatenga comprenant trois provinces, Lorum, Yatenga, et Zandoma, et pénètre même, hors de sa zone naturelle d'influence, dans les provinces comme le Kadiogo, le Kourittenga, l'Oubritenga et le Bam. Au total, Sôore entretient dixneuf comités départementaux de rédaction, des centaines de clubs de lecture et le titre est disponible dans les bibliothèques départementales de la province. Il en est de même pour Laabaali qui couvre non seulement tout le Gulmu mais les régions voisines du Niger, du Bénin et du Togo.

Les titres en langues nationales clairement destinés à un public provincial alphabétisé, très dispersé et fortement présent en milieu villageois, connaissent des problèmes de distribution spécifiques ${ }^{1}$. Sôore organise sa distribution par l'intermédiaire de ses correspondants disséminés dans les provinces du Yatenga et du Lorum. Ces derniers conservent 25 francs CFA par exemplaire vendu. Le journal est écoulé presque dans sa totalité mais les pertes dues au problème du recouvrement sont importantes. Ainsi, en 1996, Sôore a perdu 1.400.000 francs CFA et, en 1997 et 1998, 1.500.000 francs CFA en recouvrement non

1 Sur cette question, cf. S. T. Balima et M. S. Frère in Médias et Communications sociales au Burkina Faso, L'HARMATTAN, Paris, 2003. 
réalisé. Il est des mois où, sur les 2.500 exemplaires emportés par les correspondants, seul le produit d'une centaine revient effectivement au journal. La diffusion des journaux en milieu rural souffre aussi des aléas du calendrier de la production agricole. Sôore observe une baisse de ses ventes pendant la saison des pluies, où les paysans sont occupés dans leurs champs. Par contre, la période froide (décembre-janvier) est très faste pour le journal. Laabaali, qui est une émanation de l'ONG Tin Tua, tire avantage de cette parenté dans sa distribution. En effet, tous les services de Tin Tua, répartis dans les villages, sont abonnés au journal et de façon assez massive pour pouvoir l'exploiter dans leurs activités respectives. Laabaali a également mis sur pied un système de distribution assez performant en équipant ses correspondants de bicyclettes et en leur laissant 10 francs CFA par journal vendu. Lors des missions d'autres services de Tin Tua dans les villages, le bilan est dressé et de nouveaux exemplaires peuvent ainsi être acheminés à chaque déplacement sur le terrain.

La question des langues ne peut être réduite à des considérations purement arithmétiques. Elle couvre le vaste champ des enjeux politiques, culturels et historiques.

\section{Contraintes organisationnelles et considérations sociopolitiques dans l'utilisation des langues burkinabé dans les médias}

Les contraintes organisationnelles au sein des médias influent sur l'utilisation des langues nationales. Une contrainte fondamentale est celle de la place dont peuvent disposer ces langues plurielles dans une édition ou dans une journée pour traiter d'un sujet social ou d'actualité. Ces modalités de fonctionnement à la fois structurelles et rédactionnelles sont des contraintes qui limitent la portée de l'utilisation des langues de notre terroir. Ces contraintes deviennent des éléments structurant le discours des journalistes et des médias, faute de temps ou d'espace suffisants, faute de vocabulaire approprié, faute aussi d'intérêt activement exprimé par nos masses souvent confrontées au mal de l'ignorance : ignorance de leurs droits civiques, de leur citoyenneté, de leur capacité réelle à influer sur le cours de l'histoire nationale.

Dans nombre de stations de radio qui recourent aux langues nationales pour traiter l'information d'actualité, on constate une traduction du français vers ces langues avec des distorsions parfois majuscules. Le texte de base des reformulations est souvent une synthèse de l'actualité 
nationale fondée sur la communication institutionnelle des pouvoirs publics.

La traduction du texte original en diverses langues donne lieu à des improvisations multiples qui fécondent le même événement. Ces traducteurs, passés maîtres dans l'art du compte rendu imagé, comblent assez aisément l'insuffisance notoire de ces textes de synthèse préparés et rédigés par les rédacteurs en français. De sorte que les auditeurs ne sont plus devant la nouvelle mais dans la nouvelle. Cette participation au récit des événements que suscite le parler local aboutit à une sorte de « dramatisation », de défiguration et de gonflement de l'information de la part des présentateurs. La conséquence pratique de cette situation est que les différents groupes linguistiques ne reçoivent pas la même information dans les mêmes termes. Il peut donc en résulter des différences d'interprétation considérables sinon contradictoires. La langue constitue non seulement l'une des composantes essentielles de la culture, mais également elle montre que, compte tenu du rapport entre les deux, le traducteur doit être bilingue et biculturel, car les différentes langues ne perçoivent pas la réalité de la même façon. Les liens qui fondent le couple "langue-culture" sont si forts que Richard (1998:151) note que « qui change de langue doit changer de culture ${ }^{1}$ ».

Nos traducteurs ne s'embarrassent pas de ces considérations. L'exagération par le biais des hyperboles est devenue un procédé courant chez nos animateurs en langues locales, en particulier sur les ondes de certaines radios privées où l'on voit apparaître de nouvelles vedettes de l'information.

La place limitée accordée aux langues nationales dans les grands médias nationaux est dictée par des considérations géoculturelles dont les conséquences identitaires sont réelles. Il y a, de fait, une lutte interne aux langues nationales dont les enjeux sont la possibilité d'avoir une ascendance sur les autres parlers du pays. Or, cette lutte larvée n'est pas sans effet sur le discours du journaliste ou de l'animateur qui souvent, se refuse à traduire les témoignages en langues mooré, jula ou fulfuldé en langue officielle. En s'abstenant de traduire les points de vue exprimés en langues nationales, les médias minimisent les locuteurs et se substituent facilement à ces derniers pour contrôler ce qui est dit au sujet de l'objet rapporté. Ce faisant, on voit rarement des témoignages

1 Lalbila Aristide Yoda, La traduction médicale du français vers le mooré et le bisa : un cas de communication interculturelle au Burkina Faso, RUG, Grönigen, 2005, p. 6. 
en langues nationales dans les productions médiatiques sur l'actualité, considérée comme la chasse gardée des francophones à part entière sinon des francophones à part évoluant en dehors du pays réel.

Une autre caractéristique de ces éditions en langues nationales, est la rareté des témoignages vivants et directs, d'interviews de personnalités en langues nationales, excepté quelques parenthèses de circonstance. Tout se passe comme si la frange des locuteurs en langues du pays n'avait droit qu'à un traitement de seconde zone. Ainsi, les sources issues du terroir ou du pays réel sont des sources périphériques.

Du même coup, les quelques témoignages en langues nationales, quand ils sont traduits en français, sont approximatifs ou escamotés. Cette pratique réside dans la gestion des sources, importante pour tous les sujets dont traitent les journalistes.

Ainsi, les médias sont des acteurs qui construisent les représentations linguistiques, les conceptions, informations et repères constitutifs de la symbolique sociale et les mettent en discours, en normes et en valeurs.

«Autant que leur rhétorique, c'est l'ensemble du vocabulaire utilisé dans les médias qui contribue à fixer les cadres de référence en dehors desquels aucun débat n'est jugé possible. L'activité sociale des médias consiste à délimiter le nombre de définitions permises. ${ }^{1}{ }^{~}$

Au sujet des langues, les médias fixent leur cadre, délimitent leur espace et leur temps, définissent leurs hiérarchies. Cette symbolique sociale portée par les médias ne reflète pas les opinions et expériences des Burkinabè, mais elle s'effectue comme une donnée normative qui agit à travers une spirale du silence face aux langues dominantes au sein de notre communauté nationale. Ainsi, les trois langues véhiculaires évoluent en se transformant en langues dominantes.

En réalité, l'activité médiatique n'est pas neutre dans la façon dont les langues nationales sont utilisées; elle s'inscrit dans les mutations profondes et structurantes d'une démocratie linguistique et en traduit les lignes de force. Cette réalité n'est pas sans conséquence sur la façon dont les médias peuvent rendre compte des événements dans les langues minoritaires qui, dans les faits, ont tendance à être marginalisées.

Au regard de l'utilisation du français dans les médias, toutes les langues locales ont la même position sociale. Cette différence de fait entre le français et les autres tient au fait que les langues locales n'ont

1 Paul Beaud, La société de connivence, Aubier-Montaigne, Paris, 1984, p. 292. 
pas acquis le statut culturel et social de cette langue étrangère indépendamment de laquelle elles se sont développées, même si, aujourd'hui, on constate une certaine influence de la langue officielle sur le registre de nos parlers locaux à travers certains emprunts.

La classification en langues privilégiées et en langues démunies découle non pas de considérations linguistiques mais de critères politiques. En d'autres termes, l'idiome parlé par la classe la moins privilégiée reste défavorisé dans les productions médiatiques. Ceci parce que la langue française est une clé sociale qui confère des pouvoirs exorbitants à ses locuteurs. La question est complexe et Staline a pu dire en 1950 que « la langue est un instrument qui sert également toutes les classes de la société et manifeste à leur égard une totale indifférence. Autrement dit, il n'y a pas de langue de classe mais un usage de classe de la langue ${ }^{1} »$.

Ce point de vue de Staline nous parait discutable et ne s'accommode, à notre avis, que de situations unilingues. Dans le cas du plurilinguisme, nous avons une langue dominante (le français) que les élites parlent par besoin et intérêt, et des langues dominées (celles des indigènes). Ces élites ou plutôt ces locuteurs en langue française n'excèdent pas $10 \%$ de la population burkinabè, si l'on en croit les chercheurs Issa Diallo et Lamine Sanogo du CNRST ${ }^{2}$. Ces derniers ont relevé que le schéma sociolinguistique du pays consacre la domination de la langue française comme langue de traitement de l'information d'actualité qui s'accapare tous les statuts, loin devant le mooré, le jula et le fulfuldé. Pourtant ces trois langues nationales couvrent, à elles seules, la quasitotalité des besoins de communication sociale du pays.

Malgré ce rapport de force politique en faveur de la langue française, on peut noter une évolution quantitative des langues nationales au niveau de la télévision et des radios locales dont l'origine remonte en fait aux idéaux de la Révolution d'août 1983.

En effet, cette Révolution d'août 1983 a favorisé l'essor des langues nationales à partir de l'implantation de stations locales ou régionales. Ce qui a eu pour conséquence immédiate de décupler le volume hebdomadaire des langues nationales sur le réseau radiophonique national. Il en est de même sous la $I V^{e}$ République avec l'introduction à la télévision des principales langues du pays, utilisées pour l'information et l'éduca-

\footnotetext{
J. Staline cité par S. T. Balima, ibid.

2 Issa Diallo et Lamine M Sanogo, Problématique de l'information en langues nationales, Colloque de janvier 2004, Université de Ouagadougou.
} 
tion, consacrant une certaine démocratisation de ce moyen de communication, naguère décrit comme un class-médium. Et aujourd'hui, la télévision nationale utilise au moins une dizaine de langues nationales dans ses programmes d'information hebdomadaires.

Si l'on observe la situation au niveau national, on relève des inégalités flagrantes entre les langues nationales dans leur utilisation sur les antennes des radios et télévisions publiques, et sur celles des stations privées du type commercial ou communautaire. Autrement dit entre les langues nationales, il y a les privilégiées et les défavorisées dans le système médiatique

A première vue, la répartition inégale des volumes horaires entre les langues du pays peut être comprise comme résultant seulement d'un phénomène sociologique, le mooré, le jula et le fulfuldé étant des langues véhiculaires dominantes.

Si pour ces trois principales langues on peut justifier leur prédominance relative sur les autres par l'importance numérique des populations qui communiquent avec ces langues, on note des disparités dans l'utilisation des 56 autres langues dont certaines ne sont pas encore écrites sur des supports de grande diffusion, ni entendues sur les ondes et des moyens de diffusion modernes.

\section{Conclusion}

La place des langues nationales dans les médias reste relativement faible. Visiblement leur promotion se heurte à des pesanteurs diverses mais celles-ci ne sont pas insurmontables.

La réorganisation de la distribution des journaux en langues nationales passe certainement par une réelle motivation du personnel permanent et des collaborateurs. Une solution de pis-aller semble avoir été choisie par Sôore qui fait à la fois de ses rédacteurs départementaux ses distributeurs dépositaires vendeurs, une confusion des fonctions qui peut être fort dommageable. Ici encore, la stratégie de Laabaali, qui consiste à distinguer nettement les fonctions de rédaction et de vendeur, est réaliste.

Il faut donc aux promoteurs de cette presse une vision dans la stratégie de développement et une grande rigueur dans l'organisation et la gestion. Ces publications en langues locales sont, non seulement indispensables à la post-alphabétisation mais aussi à la sauvegarde de la communication participative pour le développement. 
Dans l'audiovisuel, les langues nationales permettent non seulement de prolonger la tradition orale mais aussi de créer une nouvelle dynamique sociale par l'implication du plus grand nombre de nos acteurs, ceux qui sont exclus de la pratique francophone. 
